\title{
The long and winding road of chest radiography for tuberculosis detection
}

\author{
Cecily Miller ${ }^{1}$, Knut Lonnroth², Giovanni Sotgiu $\mathbb{1}^{3}$ and Giovanni Battista Migliori $\mathbb{1}^{4}$ \\ Affiliations: ${ }^{1}$ Dept of Medicine, University of California San Francisco, San Francisco, CA, USA. ${ }^{2}$ Global TB \\ Programme, World Health Organization, Geneva, Switzerland. ${ }^{3}$ University of Sassari Medical School, Dept of \\ Biomedical Sciences, Sassari, Italy. ${ }^{4}$ World Health Organization Collaborating Centre for Tuberculosis and \\ Lung Diseases, Fondazione S. Maugeri, Care and Research Institute, Tradate, Italy.
}

Correspondence: Cecily Miller, Dept of Medicine, University of California San Francisco, 1001 Potrero Avenue, San Francisco, CA 94110, USA. E-mail: cecily.millerducsf.edu

@ERSpublications

Chest radiography, an old tool for TB detection, is back at the forefront of the global effort to end TB http://ow.ly/92wI30aCxpq

Cite this article as: Miller C, Lonnroth $\mathrm{K}$, Sotgiu G, et al. The long and winding road of chest radiography for tuberculosis detection. Eur Respir J 2017; 49: 1700364 [https://doi.org/10.1183/13993003.00364-2017].

\section{Introduction}

The aspiration to ensure early diagnosis for all people with tuberculosis (TB) [1], combined with recent advancements in digital technology, has brought chest radiography back to the forefront for TB detection. Once a mainstay for TB control in the community, but discouraged once bacteriology became the standard for TB diagnosis, chest radiography has recently come back into favour for its ability to detect TB early, often before the onset of clear symptoms. In addition, the advent of digital radiography has improved the quality and safety of the procedure and has led to possibilities such as telemedicine and computer-aided reading and detection.

\section{History of chest radiography for TB diagnosis}

Chest radiography has a long history of use in TB care and control. Over a century ago it was used to detect patients with active disease for management and infection control, often in sanatoria. When antibiotics that could cure the disease became available in the late 1940s, radiography played a central role in identifying those eligible for treatment. Large-scale screening campaigns using radiography to detect TB began in industrialised countries in the early 20th century, when TB was still a leading cause of death in those settings. Mass radiography screening campaigns were commonplace from the 1930s to the 1960s and were conducted everywhere, from major cities to isolated communities [2]. Mobile X-ray units in trolley cars, buses, railroad cars, aeroplanes and boats were used so that hard-to-reach populations could access radiography services (figure 1). Celebrities even rallied to support the cause and encourage people to be screened for TB (figure 2). Many of these mass screening campaigns were successful at detecting a large pool of prevalent TB cases, and surveys of follow-up campaigns often appeared to show significant reductions in the burden of this disease [2]. However, the actual impact on TB transmission of such campaigns remains uncertain [3], as other factors, including gradually improved access to high-quality treatment as well as socioeconomic improvements, also contributed to the declining TB burden [4].

Moreover, the campaigns were expensive and logistically difficult to implement, and required a significant investment of manpower from highly skilled technicians. Once the burden of TB was significantly reduced in industrialised countries, in the 1960s-1970s it no longer made sense to invest in them. Meanwhile, in developing countries that still bore a heavy burden of TB, mass screening with radiography was proving 
FIGURE 1 Public Health Service mobile chest radiography van with a line of men waiting their turn. Source: US National Library of Medicine.

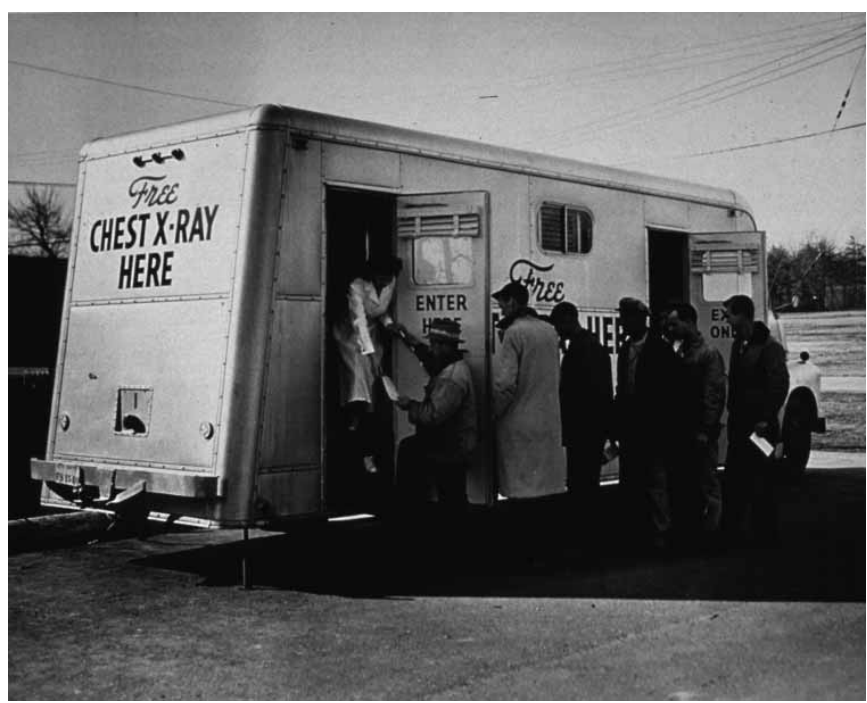

too expensive and logistically challenging to be sustainable. There was also a growing consensus that chest radiography was not sufficiently reliable to be used to establish a diagnosis of TB, given its intra- and inter-reader variability and lack of specificity for TB $[5,6]$. Thus, there was a shift in policy away from using radiography to detect TB towards case detection in the primary care setting, based on symptom screening combined with the use of bacteriological investigation of sputum to establish a diagnosis. In 1974 the World Health Organization (WHO) issued a recommendation to abandon "indiscriminate TB case-finding by mobile mass radiography" [7].

FIGURE 2 A collage of well-known celebrities who gave publicity and support to a chest radiography campaign in Los Angeles, California in 1950. Source: US National Library of Medicine.

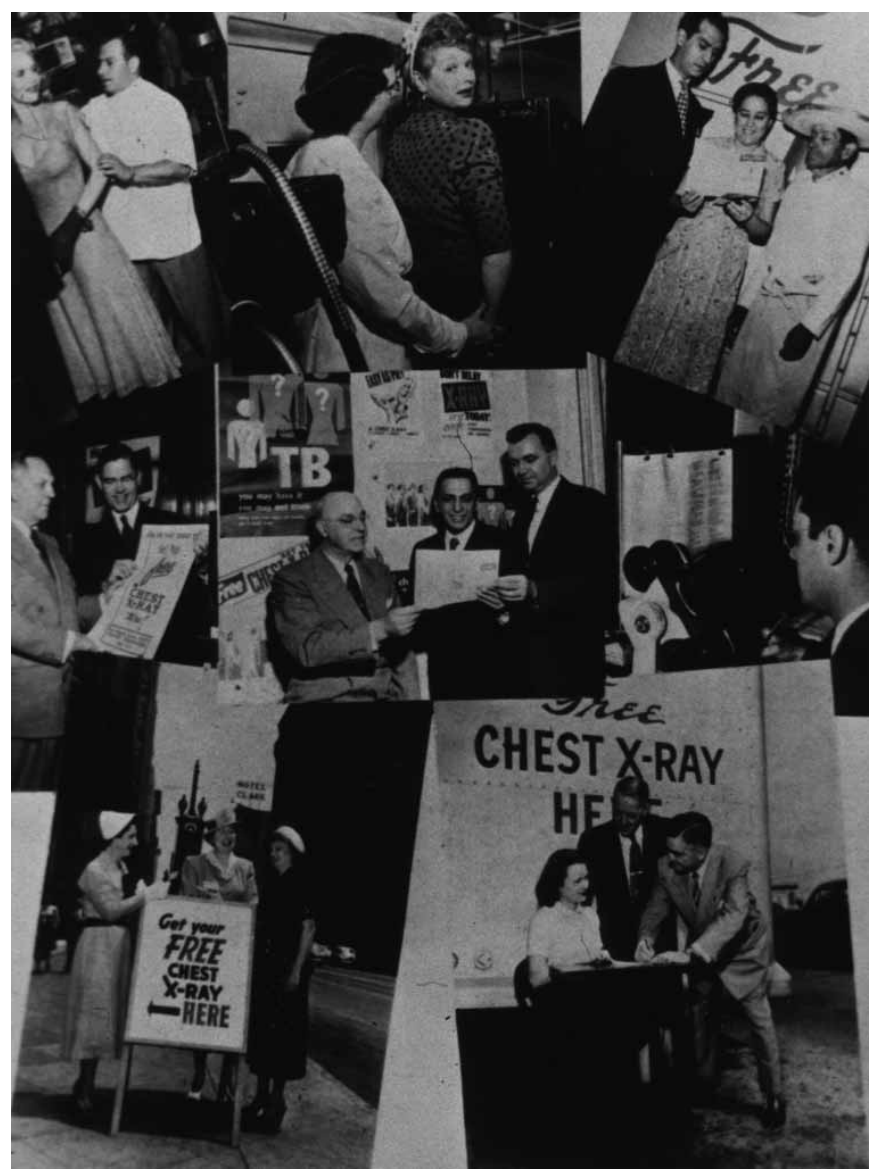




\section{Renewed interest in chest radiography for TB screening}

However, the use of chest radiography for investigation of TB has continued in the clinical setting throughout the past few decades in developed and developing countries alike, even as it fell out of favour in national and global public health policy (figure 3). This is in part due to the fact that chest radiography is not only used for TB diagnosis but is also a valuable imaging tool for a range of lung diseases. Recently, a combination of factors has led to a resurgence of global policy in favour of chest radiography for TB detection. First, the HIV pandemic, and its associated increase in smear-negative TB, has necessitated a rethinking of the standard approach to TB diagnosis. Second, several recent prevalence surveys have demonstrated that a significant proportion of TB cases are asymptomatic, but detectable through chest radiography. Third, the global priority in $\mathrm{TB}$ detection has shifted from a focus on finding the most infectious cases of disease to aspiring to early diagnosis of all TB cases as part of the End TB Strategy [1].

New diagnostic tests for TB are now available to help achieve the goal of more complete detection of TB, such as the automated nucleic acid amplification test Xpert MTB/RIF (Cepheid, Sunnyvale, CA, USA), which greatly improves upon both the sensitivity and specificity of sputum smear microscopy, but comes at a much higher cost. Thus, there is a need to pre-screen individuals for TB evaluation to determine who should be evaluated by means of these highly accurate yet expensive tests, such that the highest possible yield of TB cases is detected in the most cost-effective manner possible. Chest radiography is an excellent tool for such screening, given its high sensitivity and lower relative cost. Accordingly, it is now starting to appear as an early screening or triage test in TB diagnostic algorithms, to identify patients for further evaluation by bacteriological methods, rather than by algorithmic means as a tool of last resort [8]. The combination of radiological and molecular tools could be key to improving early TB diagnosis in the era of the End TB Strategy.

\section{Recent technological advancements in chest radiography and TB detection}

Recent advancements in digital technology for radiography have led to improvements in imaging performance and functionality, including reduced operating costs, better image quality, lower radiation dosage, improved data storage options, and the potential for digital image transfer for telemedicine. Digital advancements have also paved the way for technological innovations in TB detection using radiography, such as computer-aided detection (CAD) software programmes. Such programmes can be used to standardise the interpretation of chest radiographic images for TB detection, reducing reader variability and error. The ability to use technology to interpret radiographic images could help in settings where there is a lack of skilled personnel. Automated programmes could also ultimately lower the costs of a chest radiograph by reducing the provider time required, which is especially important given that, in many lowand middle-income countries, the costs of chest radiography for TB detection still fall on the patient and can be prohibitively high [9].

One such programme for TB detection is highlighted in the paper by RAHMAN et al. [10] that appears in this issue, entitled "An evaluation of automated chest radiography reading software for tuberculosis screening among public- and private-sector patients." This paper evaluates CAD4TB, a software developed to assign an abnormality score ranging from 0 to 100 to a digital chest radiographic image that reflects the likelihood of tuberculosis based on the shape and texture of abnormalities observed in the image. RAHMAN et al. [10] applied CAD4TB to over 17000 patients from private providers and public clinics referred for TB evaluation in Dhaka, Bangladesh. They show that CAD4TB, using a threshold set to match the sensitivity of the radiologists in the study, could be used to triage symptomatic individuals for testing with

FIGURE 3 Chest radiographs being read in Iringa District, Tanzania. Source: Philip Hopewell.

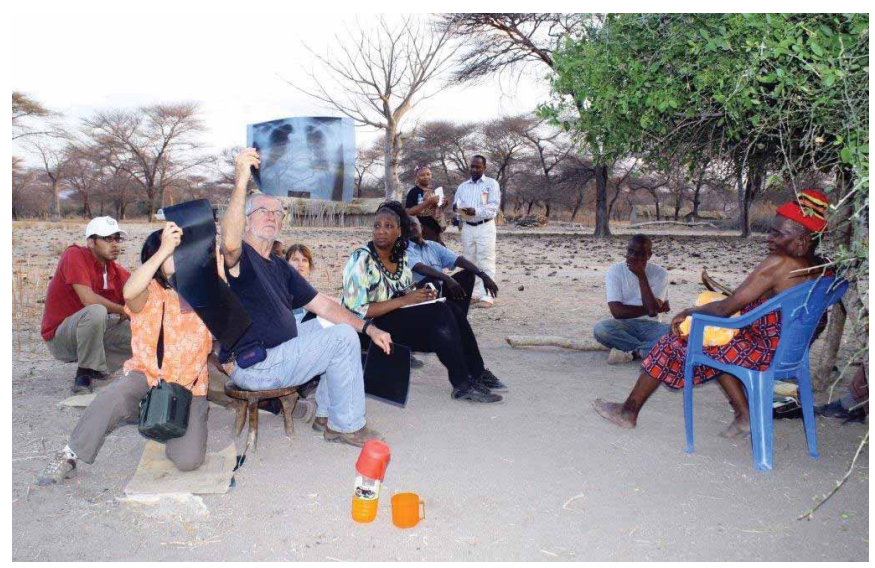


Xpert MTB/RIF, resulting in a $36 \%$ reduction in the number of Xpert tests conducted while only missing $9 \%$ of TB cases.

However, this study also shows that CAD has some way to go before it is perfected for TB detection. In this analysis, the computer programme could not match both the sensitivity and specificity of the radiologist for interpreting chest radiographic images, overall and across all subgroup analyses. Moreover, $\mathrm{CAD} 4 \mathrm{~TB}$ ultimately proved more expensive than the radiologist service. This evaluation also showed the variability of CAD4TB performance across different subgroups of patients, and the complexity in choosing the appropriate threshold score as a cut-off for referral for further evaluation. These issues all require further study before the software can be utilised on a large scale.

A global consultation meeting held by the WHO in September 2016 concluded that there is currently insufficient data on the accuracy and performance of CAD programmes for $\mathrm{TB}$ to issue definitive guidelines [8]. At the time, the evidence was limited by the small number of studies and the lack of generalisability of the available data. The WHO and partners are developing advice on key research questions and appropriate study designs so that future research on CAD for TB detection can help inform global guidance. Such research questions include evaluating the added value of CAD in the diagnostic pathway, the cost-effectiveness of CAD compared with human readers, and the implementation challenges involved in using CAD in different settings.

\section{Conclusion}

Just as in other sectors of healthcare, advancements in digital technology are rapidly expanding the potential contribution of chest radiography to TB detection globally. Further research such as the evaluation found in this month's issue will be required to determine how CAD can best be used to improve TB detection across a variety of settings. More broadly, there is a need for a comprehensive view of the contribution that chest radiography can make to support the control and elimination of TB. It is essential that we utilise all available tools, old and new, to achieve the End TB Strategy's aim of ending the global TB epidemic in this century.

\section{References}

Uplekar M, Weil D, Lonnroth K, et al. WHO's new End TB Strategy. Lancet 2015; 385: 1799-1801.

2 Golub JE, Mohan CI, Comstock GW, et al. Active case finding of tuberculosis: historical perspective and future prospects. Int J Tuberc Lung Dis 2005; 9: 1183-1203.

3 Kranzer K, Afnan-Holmes $\mathrm{H}$, Tomlin $\mathrm{K}$, et al. The benefits to communities and individuals of screening for active tuberculosis disease: a systematic review. Int J Tuberc Lung Dis 2013; 17: 432-446.

4 Lonnroth K, Jaramillo E, Williams BG, et al. Drivers of tuberculosis epidemics: the role of risk factors and social determinants. Soc Sci Med 2009; 68: 2240-2246.

5 Springett V. Results of the study on x-ray readings of the Ad Hoc Committee for the study of classification and terminology in tuberculosis. Bull Int Union Tuberc Lung Dis 1968; 41: 107-109.

6 Springett V. Results of the international study on x-ray classification. Conclusions. Bull Int Union Tuberc Lung Dis 1968; 41: 125-129.

7 World Health Organization. WHO Expert Committee on Tuberculosis, Ninth Report. Technical Report Series no. 552. Geneva, WHO, 1974

8 World Health Organization. Chest Radiography in Tuberculosis Detection - Summary of Current WHO Recommendations and Guidance on Programmatic Approaches. Geneva, WHO, 2016.

9 Pedrazzoli D, Lalli M, Boccia D, et al. Can tuberculosis patients in resource-constrained settings afford chest radiography? Eur Respir J 2017; 49: 1601877.

10 Rahman MT, Codlin AJ, Rahman MM, et al. An evaluation of automated chest radiography reading software for tuberculosis screening among public- and private-sector patients. Eur Respir J 2017; 49: 1602159. 